\title{
LANDAU CAVITIES AT MAX II
}

\author{
Å. Andersson, M. Eriksson, M. Georgsson, MAX-lab, Lund, Sweden
}

\section{Abstract}

Passive Landau cavities have been built, installed and taken into regular operation in the MAX II ring. The purpose of the Landau damping system is twofold: to decrease the energy spread of the beam induced by coupled bunch oscillations and to increase the Touschek life-time. The cavities are of the pillbox type operated at $1500 \mathrm{MHz}$, the third harmonic of the main RF. This paper describes the design and operational results of the cavities.

\section{INTRODUCTION}

The MAX II ring is a $1.5 \mathrm{GeV}$ third generation synchrotron light source in Lund, Sweden [1]. Target specifications and ring parameters are given in Table 1. All the target specifications had been met, except that for the energy spread of the electron beam. This increased energy spread is due to coupled bunch oscillations and would deteriorate the performance of the undulators when used at higher harmonics. A feed-back or a HOM damping system has been considered, but this should imply a short Touschek lifetime. In this situation a Landau cavity system was chosen to solve the problem. Such a system should both damp the coupled bunch instability, and prolong the Touschek life-time. The damping should be provided by the nonlinear RF waveform, and the increased lifetime should come from the reduced gradient at the bunch position, giving a longer bunch with less density.

At MAX II the Landau cavities are used passively, i.e. they are excited by the electron beam itself. Passive Landau cavities have been used before, and at MAX I there is one working under certain conditions [2,3]. Landau cavities have successfully been used elsewhere e.g. $[4,5]$.

Table 1: The target specifications of MAX II.

$\begin{array}{lll}\text { Maximum energy } & E & 1.5 \mathrm{GeV} \\ \text { Current } & I_{c} & 200 \mathrm{~mA} \\ \text { Lifetime } & \tau & 10 \mathrm{~h} \\ \text { Fundamental RF } & f_{f} & 500 \mathrm{MHz} \\ \text { Emittance } & \varepsilon & 8.8 \mathrm{nmrad} \\ \text { Natural energy spread } & \sigma / E & 0.7 \cdot 10^{-3} \\ \text { Momentum compaction factor } & \alpha & 0.004 \\ \text { Dispersion in straight sections } & \eta & 0.13 \mathrm{~m} \\ \text { Coupling } & \varepsilon_{\mathrm{y} /} / \varepsilon_{\mathrm{x}} & 10 \%\end{array}$

\section{DESIGN CONSIDERATIONS}

A passive cavity system was chosen due to its simplicity, compared to an active one. Furthermore, a third harmonic system, at $1.5 \mathrm{GHz}$, was chosen, since a higher frequency system would have suffered from a low shunt impedance, with the given vacuum pipe dimensions. The design criteria were the following:

1. The overall RF voltage derivative should be close to zero at the position of the bunches.

2. The maximum power taken from the beam should not exceed $10 \mathrm{~kW}$.

3. The fundamental $500 \mathrm{MHz}$ RF voltage is $0.8 \mathrm{MV}$.

4. The operating circulating current range should be $0.1 \mathrm{~A} \leq I_{c} \leq 0.2 \mathrm{~A}$.

5. The coupled bunch instability should be suppressed

6. The beam should be Robinson damped.

In the MAX-II case 1-4 requires [6] that $R_{\mathrm{sh}}>7.5 \mathrm{M} \Omega$ (linac definition). This shunt impedance also provides Robinson damping. Measurements [6] on the real pill-box type cavities showed a shunt resistance of $2 \mathrm{M} \Omega$, hence four of them had to be installed.

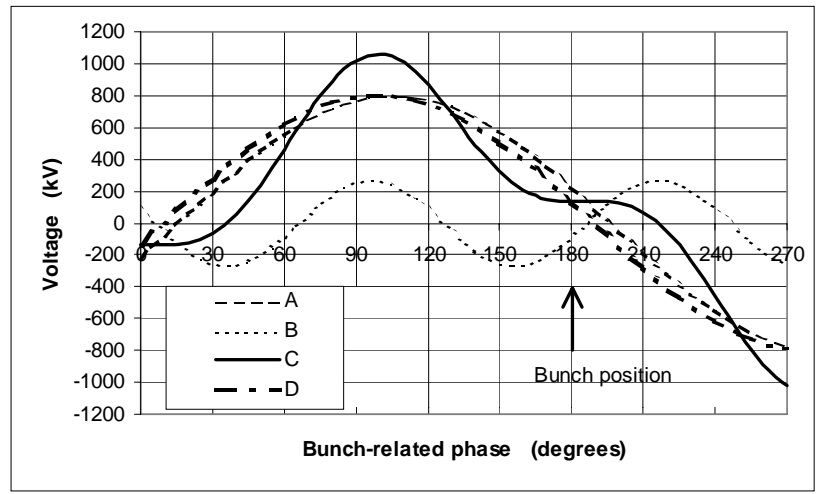

Figure 1: Design voltages in the double RF system. A: main cavity voltage with the passive system tuned, B: voltage in the passive cavity system, C: total voltage, $\mathrm{D}$ : main cavity voltage with the passive system detuned.

The different voltages in the double RF system are shown in Fig. 1. To reach zero gradient, each Landau cavity should roughly keep an RF level equal to $1 / 12$ of the main RF voltage.

\section{MEASUREMENTS}

First the plunger position for resonance was found for each cavity one by one, operating at low currents. Now each probe signal could be calibrated towards the Landau cavity field, by $U=I_{c} \cdot R_{s h}$. The probe signals were then 
used to determine the cavity fields during operation. After the calibration, all four cavities were operated in parallel.

Measurements of energy spread and bunch length were performed during a series of fillings of MAX II. A certain $\mathrm{RF}$ voltage level in the Landau-cavities were chosen and kept constant, by plunger tuning, during the current decay. Due to out-gassing problems in the main cavity, its fields were maximised to $U_{f}=500 \mathrm{kV}$ during the experiments. Careful measurements of the synchrotron frequency gave precise indication on the total voltage slope at the bunch position.

\subsection{Electron beam energy spread}

The energy spread was determined from beamsize measurements [7]. At minimum coupling, less than $1 \%$ in this case, the horizontal beam emittance is least sensitive to coupling variations. Thus, at low currents the horizontal beamsize is determined and compared to the theoretical value

$$
\sigma_{x}=\sqrt{\varepsilon_{x} \beta_{x}+\left(\eta \sigma_{e} / E\right)^{2}}
$$

where $\sigma_{e} / E=0.7 \cdot 10^{-3}$ is the natural energy spread, and $\beta_{x}$ is the horizontal beta-function at the observation point. This was done for differing optics which gave consistent result for the natural energy spread. By measuring $\sigma_{x}$ at higher currents the energy spread is derived from (1). With detuned cavities the energy spread is $4-5 \cdot 10^{-3}$ due to the coupled bunch oscillations, but with tuned Landau cavities the energy spread decreased down to $1-2 \cdot 10^{-3}$. Fig. 2 shows a setting with Landau cavities tuned to $\mathrm{U}=18 \pm 2 \mathrm{kV}$.

It was found that the most pronounced decrement in energy spread, down to $2 \cdot 10^{-3}$, was reached already at 11 $\mathrm{kV}$ in each Landau cavity. With further tuning $\sigma / E$ $=1.1 \cdot 10^{-3}$ could be achieved. At the time of measurements the zero gradient case, requiring $42 \mathrm{kV}$ in each cavity, could not be reached due to out-gassing problems in the Landau cavities.

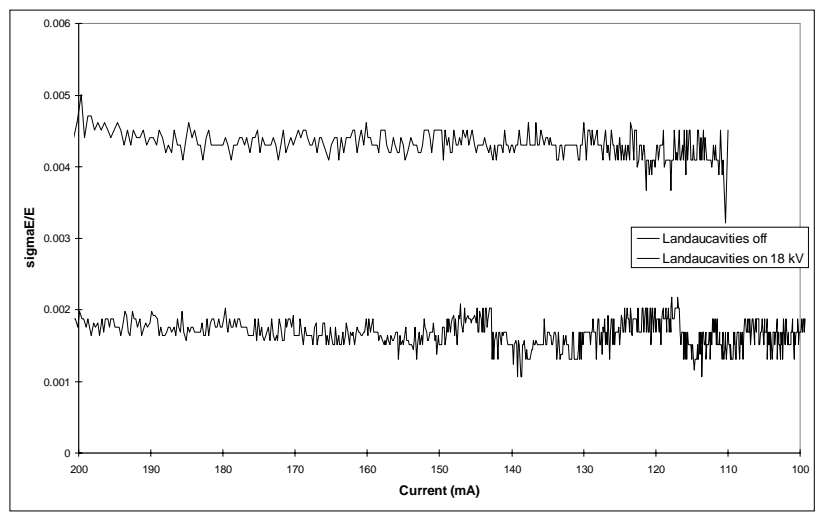

Figure 2: The beam energy spread versus beam current with and without Landau cavities.
At the undulator beamline 411 spectra at higher harmonics were taken with and without the Landau cavities engaged [8]. Fig. 3 shows a typical result.

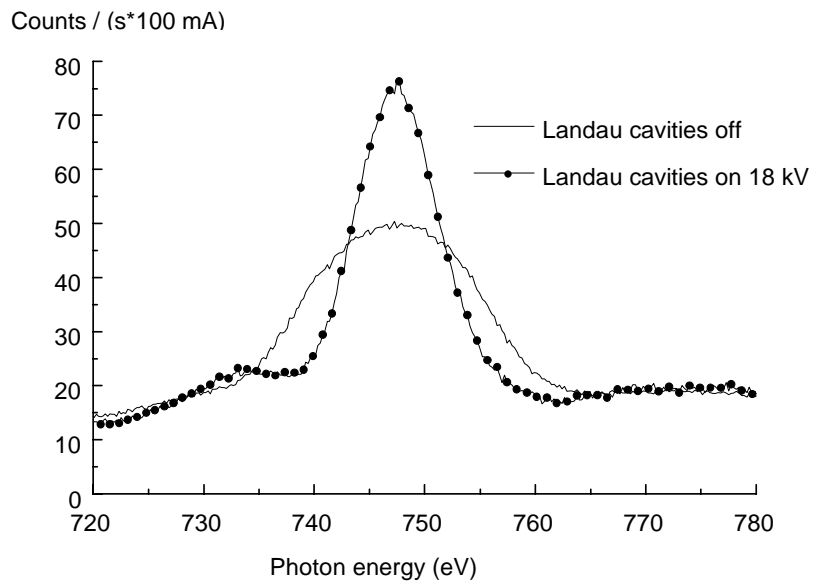

Figure 3: The effect of the Landau cavities on a high harmonic of undulator 411.

\subsection{Bunch length}

Bunch length measurements were performed using an optical sampling oscilloscope (OSO) [7]. However, the OSO is a time averaging instrument when triggered by the RF clock, and the measurements give no information on actual bunch length in the case we have coupledbunch oscillations. The natural RMS bunch length is 24 ps, at $U_{f}=500 \mathrm{kV}$. But due to strong coupled bunch oscillations we measure it to $160-170 \mathrm{ps}$ at ring currents above $100 \mathrm{~mA}$ (Fig.3.3). These numbers indicate an energy spread which is seven times higher than the natural one, considering the bunch length given by

$$
\sigma_{t}=\alpha / \Omega_{s} \cdot \sigma_{e} / E
$$

where $\sigma_{e} / E$ is the sum of coherent and incoherent energy oscillations. With the Landau cavities a bunch length of about $80 \mathrm{ps}$ is measured at $18 \mathrm{kV}$ excitation. Thus, the energy oscillations are effectively damped. However, again considering equ. (2), keeping in mind that at the low excitation levels used in the Landau cavities the quadratic potential term is still dominant, with the measured $\Omega_{s}=2 \pi \times 14 \mathrm{kHz}$ one arrives at $\sigma_{e} / E=1.8 \times 10^{-3}$. This is in very good agreement with the previously measured energy spread. As can be seen from Fig. 4, going either to higher or lower excitation leaves the measured bunch lengths quite unaffected, since then both energy spread and synchrotron frequency either increase or decrease. 


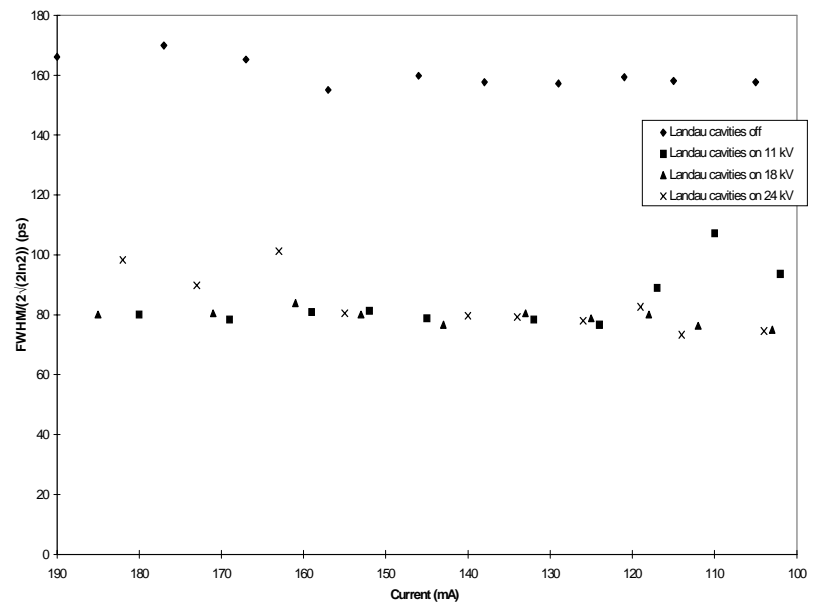

Figure 4: Bunch lengths, as measured with the OSO, with and without Landau cavities.

\section{ROUTINE OPERATION}

In normal SR production mode an emittance coupling of $10 \%$ is used. Presently, an excitation level of $18 \mathrm{kV}$ in each Landau cavity is used, giving an energy spread of $1.8 \cdot 10^{-3}$. The effect of the Landau cavity system on the beam lifetime is dramatic as can be seen from Fig. 5. The landau damping ceases at around $70 \mathrm{~mA}$. However, at routine operation the ring is now filled with $250 \mathrm{~mA}$, which means that the current does not decay below 100 $\mathrm{mA}$ even after one day. This admits injection only once per day, in spite of the fact that the main cavity field level is only $500 \mathrm{kV}$.

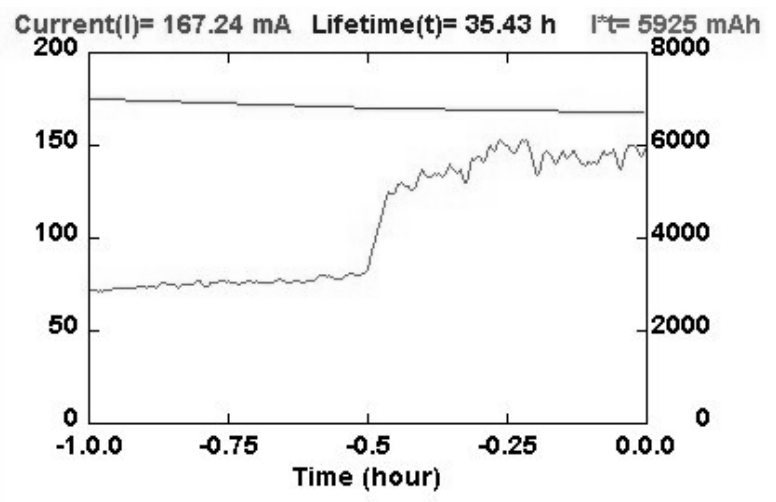

Figure 5: Beam current, and beam current times beam lifetime, versus time. The Landau cavities are tuned to $18 \mathrm{kV}$, at $-0.5 \mathrm{~h}$. The measurement is done with $10 \%$ emittance coupling..

\section{CONCLUSIONS}

The passive Landau cavity system is very effective already at excitation levels far below the design level giving zero gradient at the bunch position. The beam energy spread can easily be diminished from $5 \cdot 10^{-3}$ to $2 \cdot 10^{-3}$ for currents from $250 \mathrm{~mA}$ down to $70 \mathrm{~mA}$, merely by keeping a constant excitation level in the cavities. No limitation for higher currents has been seen, while at lower currents the Landau damping ceases. The beam lifetime increases dramatically as the Landau cavities are tuned in. However, it is quite evident that the Landau cavity system, when operated as described, not is killing all coherent energy oscillations. By tuning the cavities closer to resonance the energy spread can be diminished further, down to $1.1 \cdot 10^{-3}$, leaving the Touschek lifetime fairly constant. At these excitation levels, $\sim 30 \mathrm{kV}$, outgassing problems presently start to appear in the Landau cavities, and the target of zero gradient at the bunch position has therefore not yet been reached. The question whether the natural energy spread may be reached, thus still remains open.

\section{REFERENCES}

[1] ̊. Andersson et al, "The MAX II synchrotron radiation storage ring", Nucl. Instr. and Meth. A 343 (1994) 644-649.

[2] A. Andersson, "A Landau Cavity at MAX", Max publications (NTMX-7009) 1988.

[3] R. A. Bosch, C. S. Hsue, "Suppression of Longitudinal Coupled-bunch Instabilities by a Passive Higher Harmonic Cavity", Particle Accelerators, 1993 , vol. 42 pp. 81-99.

[4] J. Keane et al, "Bunch Lengthening Control using the Fourth harmonic Cavity in the VUV ring", 1989 IEEE Part. Acc. Conf., pg. 138.

[5] K. J. Kleman, "A Bunch Lengthening RF Cavity for Aladdin", 1995 IEEE Particle Accelerator Conference, pg. 1785.

[6] M. Georgsson et al, "Landau Cavities at MAX II", (accepted for publication in NIM A).

[7] A. Andersson, "Electron beam profile measurements and emittance manipulation at the MAX-laboratory", ISBN 91-628-2686-7.

[8] A.. Andersson et al, "Electron beam characterisation by undulator radiation", these proceedings. 